\title{
Gastroesophageal reflux in preterm infants. Neonatal myths versus evidence-based medicine
}

Routine work at the neonatal intensive care unit (NICU), a lack of updated information, and the distress caused by seeing a sick infant lead us to the error of trying to establish a certain diagnosis of gastroesophageal reflux (GER) in preterm infants by means of an unnecessary accumulation of ancillary tests in a path of therapeutic obstinacy that takes to overprovision. The same scenario is observed when medicines that do not meet therapeutic goals are prescribed, and this is associated with unwanted effects that may be lifethreatening for the patient.

So far, in 2018, two documents have been published by the American Academy of Pediatrics, ${ }^{1}$ and by the North American Society for Pediatric Gastroenterology, Hepatology, and Nutrition and the European Society for Pediatric Gastroenterology, Hepatology, and Nutrition, ${ }^{2}$ respectively. Both documents provide details on the futility of tests used at neonatal units to diagnose GER in preterm infants, as well as evidence on the lack of pharmacological response to most anti-reflux drugs, which even entail a risk for the infant.

The objective of this comment is to describe and/or expand the concepts included in the documents mentioned above.

GER was defined as the passage of gastric contents into the esophagus. GER is a universal phenomenon in the preterm infant population and, in most cases, resolves spontaneously. Most reflux episodes affect the distal esophagus, are brief and asymptomatic, and may develop as regurgitation. Pathological GER (PGER) is defined as GER that causes morbidities (restricted growth being the most important one). When an infant vomits, other causes for vomiting should be ruled out, such as gastrointestinal obstruction (e.g., hypertrophy of pylorus), infections (urinary tract infection, sepsis, meningitis), neurological diseases (e.g., hydrocephalus), urinary tract obstruction, metabolic disorders, false or induced apnea episodes.

As an example, here I analyze the case of a patient born at 36 weeks of gestational age with vomiting since the second day of life. He had a pH-metry done at the health care center, which resulted pathologic. Proton pump inhibitors (PPIs) and anti-reflux milk were indicated. Vomiting persisted and the patient failed to gain weight, so his parents got very worried and asked for a second opinion from a different physician, who indicated hydrolyzed milk due to a probable cow's milk protein allergy. The infant continued vomiting even more frequently and his general condition worsened significantly, so his parents sought care at the Department of Neonatology of a different facility in Buenos Aires. Due to persistent vomiting and looking for a cause that would justify the symptoms, a series of routine tests was ordered and neonatal diabetes was diagnosed based on a preprandial glycemia value of $640 \mathrm{mg} / \mathrm{dL}$.

GER diagnosis and management in preterm infants may be confusing and perplexing due to several controversies and myths, which I will describe below.

\section{GER is a cause of apnea of prematurity: false ${ }^{3}$}

Preterm infants have a hyperactive laryngeal chemoreflex (LCR), whose stimulation triggers apnea, bradychardia, and desaturation. This, in addition to the fact that most preterm infants have GER, led to assuming that GER may cause apnea. However, according to the studies, there is no temporal relationship between GER and apnea, and the larynx does not trigger LCR with small amounts of liquid. Finally, there is no evidence indicating that a drug treatment for GER, which promotes gastrointestinal motility and reduces heartburn, will reduce the incidence of apnea in preterm infants.

\section{The pH-metry is the best reference criterion for the diagnosis of GER: false ${ }^{4}$}

There is not enough evidence to support the use of a $\mathrm{pH}$-metry as a diagnostic method of reflux in preterm infants. This test's sensitivity and specificity have not been adequately established in this population. The test does not assess non-acid reflux. This would be because preterm infants are fed at very short intervals and the presence of milk in their stomach has a $\mathrm{pH}$ neutralizing effect. The $\mathrm{pH}$-metry does not predict the diagnosis of esophagitis and its reproducibility is suboptimal.

Esophageal multichannel intraluminal impedance (MII) is a new technique that measures electrical resistance to the flow of an electrical current between two electrodes, 
thus detecting bolus movement within the esophagus. At present, this is the most accurate method to diagnose GER. The MII allows to classify reflux into acid or alkaline, correlate symptoms and reflux, determine the height of the reflux, characterize the type of the refluxate, and measure the physical clearance of the bolus.

In spite of the advantages of the MII compared to the $\mathrm{pH}$-metry, this test has certain limitations:

1. It is not available at all health care centers.

2. Result interpretation may vary greatly.

3. No normal reference patterns have been established. ${ }^{5}$

4. Result interpretation requires a great expertise. This is a very useful test to establish a symptom correlation with both types of reflux: acid and non-acid.

\section{Drugs approved for GER treatment are effective in newborn infants: false}

$\mathrm{H} 2$-blockers, such as ranitidine, have not demonstrated to be useful in randomized studies, and have been associated with a higher incidence of enterocolitis ${ }^{6}$ and, in the long term, with a higher incidence of sepsis and death, ${ }^{7}$ due to a potential microbiota alteration. ${ }^{8}$ In relation to PPIs, randomized, double-blind studies that assessed omeprazole and lanzoprazole showed that they are ineffective to reduce GER signs. No prokinetic drug has demonstrated to reduce GER symptoms in preterm infants and they all have potential adverse effects.

\section{FINAL CONCLUSIONS}

- GER is a physiological process caused, in part, by the transient relaxation of the lower esophageal sphincter that resolves without intervention before 1 year old.

- Sleeping on the left side reduces reflux episodes, without improvement of the clinical symptoms attributed to reflux (continuous crying and/or irritability).

- Head elevation was ineffective to reduce reflux, both in the supine and the prone position. Vehicle safety seats increase reflux episodes.

- There is a mistake in the understanding of this phenomenon because, like GER, this disorder is not generally modified with an intervention and, in most cases, tends to resolve spontaneously as the preterm infant grows. Therefore, in spite of all the published evidence, most likely, many parents will continue looking for a specialist who will indicate measures and drugs that may be lifethreatening for the infant.

- Based on the controvertible effectiveness of H2-blockers and PPIs and on the evidence that these drugs increase the risk for severe infections in pediatric patients (necrotizing enterocolitis, sepsis/bacteremia, pneumonia, and gastrointestinal infections), their use should be significantly restricted and extremely cautious.

- PGER diagnosis is a true challenge in preterm infants because symptoms are non-specific and diagnostic tests ( $\mathrm{pH}$-metry and MII) have diagnostic limitations due to technical problems and interpretation difficulties in preterm infants. ${ }^{9}$

Alejandro Jenik, M.D.

Hospital Italiano de Buenos Aires alejandro.jenik@hospitalitaliano.org.ar

http:/ / dx.doi.org/10.5546/ aap.2018.eng.382

To cite: Jenik A. Gastroesophageal reflux in preterm infants. Neonatal myths versus evidence-based medicine. Arch Argent Pediatr 2018;116(6):382-384.

\section{REFERENCES}

1. Eichenwald EC, Committee on Fetus and Newborn. Diagnosis and Management of Gastroesophageal Reflux in Preterm Infants. Pediatrics. 2018; 142(1):e20181061.

2. Rosen R, Vandenplas Y, Singendonk M, et al. Pediatric Gastroesophageal Reflux Clinical Practice Guidelines: Joint Recommendations of the North American Society for Pediatric Gastroenterology, Hepatology, and Nutrition and the European Society for Pediatric Gastroenterology, Hepatology, and Nutrition. I Pediatr Gastroenterol Nutr. 2018; 66(3):516-54.

3. Abu Jawdeh EG, Martin RJ. Neonatal apnea and gastroesophageal reflux (GER): is there a problem? Early Hum Dev. 2013; 89(Suppl 1):S14-6.

4. Schurr P, Findlater CK. Neonatal mythbusters: evaluating the evidence for and against pharmacologic and nonpharmacologic management of gastroesophageal reflux. Neonatal Netw. 2012; 31(4):229-41.

5. Shin MS. Esophageal $\mathrm{pH}$ and Combined Impedance-pH Monitoring in Children. Pediatr Gastroenterol Hepatol Nutr. 2014; 17(1):13-22.

6. Guillet R, Stoll BJ, Cotten CM, et al. Association of H2blocker therapy and higher incidence of necrotizing enterocolitis in very low birth weight infants. Pediatrics. 2006; 117(2):e137-42.

7. Terrin G, Passariello A, De Curtis M, et al. Ranitidine is associated with infections, necrotizing enterocolitis, and fatal outcome in newborns. Pediatrics. 2012; 129(1):e40-5.

8. Gupta RW, Tran L, Norori J, et al. Histamine-2 receptor blockers alter the fecal microbiota in premature infants. J Pediatr Gastroenterol Nutr. 2013; 56(4):397-400.

9 Martin R, Hibbs AM. Gastroesophageal reflux in premature infants. UptoDate, May 2018. [Accessed on: July 10th, 2018].Available at: https: / / www.uptodate.com/contents / gastroesophageal-reflux-in-premature-infants. 